\title{
Impact of a telemedicine-guided, population-based, STEMI network on reperfusion strategy, efficiency, and outcomes
}

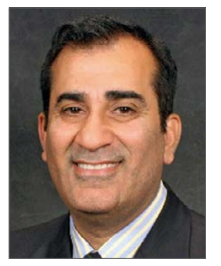

Sameer Mehta ${ }^{1}$, MD; Haytham Aboushi ${ }^{1 *}, \mathrm{MD}$; Carlos Campos², MD;

Roberto Botelho 3 , MD, PhD; Francisco Fernandez 4 , MD; Daniel Rodriguez 1 , MD;

Mario A. Torres ${ }^{1}$, MD; Daniel Vieria ${ }^{1}$, MD; Alejandra Frauenfelder ${ }^{1}$, MD; Gladys Pinto ${ }^{1}$, MD; $^{2}$

Claudia Lopez ${ }^{1}$, MD; Maria Isabel Acosta ${ }^{1}, \mathrm{MD}$

1. Lumen Foundation, Miami, FL, USA; 2. Heart Institute (InCor), University of São Paulo Medical School, São Paulo, and Hospital Israelita Albert Einstein, São Paulo, Brazil; 3. Triangulo Heart Institute and Eurolatino Medical Research, Uberlandia, Brazil; 4. ITMS, Santiago, Chile

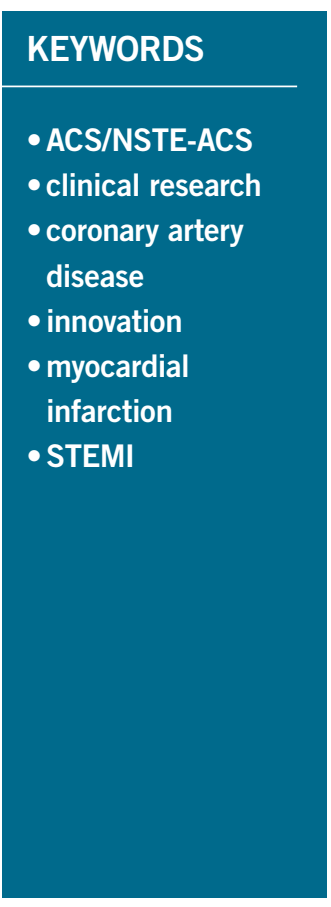

\begin{abstract}
Aims: A telemedicine-guided strategy increases the access to and efficiency of ST-elevation myocardial infarction (STEMI) networks resulting in increased access to, and reduced disparities in, acute myocardial infarction (AMI) care between rural and urban areas.

Methods and results: The Latin America Telemedicine Infarct Network (LATIN) was developed for poor and remote regions in Brazil and Colombia that lacked coordinated AMI systems of care. It strategically connects small clinics and primary care health centres (spokes) to hubs with 24/7 percutaneous coronary intervention (PCI) capability. Experts at three remote sites provide urgent electrocardiogram (ECG) diagnosis and tele-consultation for the entire network. Data from the busiest LATIN site, the Santa Marcelina Hospital in Sao Paolo, Brazil, were compared with health statistics from Sistema Unico de Saude (Brazilian Public Health System - SUS). A total of 192 centres were networked using standardised and guidelinebased protocols for AMI care. Overall, 313,897 patients were remotely screened, 3,572 AMI diagnosed (1.1\%), and 1,636 AMI urgently reperfused $(45.8 \%)$, mainly by primary PCI $(n=1,351 ; 83 \%)$.
\end{abstract}

Conclusions: In conclusion, a comparison between a pre-LATIN cohort from SUS $(1,015)$ and a LATIN cohort from Santa Marcelina Hospital $(1,247)$ revealed increased reperfusion with PCI $(65.52 \%$ vs $75.2 \%)$, increased cost $(\$ 2,037.12$ vs $\$ 2,246.40, \mathrm{p}<0.005)$, a statistically significant reduction in PCI mortality $(8.5 \%$ vs $4.3 \% \mathrm{p}<0.01)$ and a non-significant reduction in mortality overall amongst all treatment pathways $(9.69 \%$ vs $9.43 \%, \mathrm{p}=0.931)$.

\footnotetext{
*Corresponding author: Lumen Foundation, 185 Shore Dr S, Miami, FL 33133, USA.

E-mail:Haboushi10@gmail.com
} 


\section{Abbreviations}

$\begin{array}{ll}\text { AMI } & \text { acute myocardial infarction } \\ \text { CABG } & \text { coronary artery bypass graft } \\ \text { DIDO } & \text { door in door out } \\ \text { D2B } & \text { door to balloon time } \\ \text { D2N } & \text { door to needle time } \\ \text { ECG } & \text { electrocardiogram } \\ \text { ICU } & \text { intensive care unit } \\ \text { LATIN } & \text { Latin America Telemedicine Infarct Network } \\ \text { PCI } & \text { percutaneous coronary intervention } \\ \text { STEMI } & \text { ST-elevation myocardial infarction } \\ \text { TT } & \text { transfer time } \\ \text { TTD } & \text { time to telemedicine diagnosis }\end{array}$

\section{Introduction}

Telemedicine is a promising technology for managing ST-elevation myocardial infarction (STEMI), especially in developing countries where care is compromised by lack of resources, delayed patient presentation, and an absence of systems of care. Updated STEMI guidelines from the American Heart Association, American College of Cardiology, and the European Society of Cardiology emphasise the need to develop regional systems of care for primary percutaneous coronary intervention $(\mathrm{PCI})^{1,2}$. Immediate revascularisation in STEMI is the standard of care and a strategy that can be successfully applied to entire populations irrespective of individual socio-economic status ${ }^{3,4}$.

Furthermore, longitudinal studies demonstrate long-term improvements in patient outcomes when guideline-based strategies to reduce door to balloon time (D2B) are systematically adopted to create a STEMI network ${ }^{5,6}$. The American College of Physicians has made official policy and position recommendations regarding the use of telemedicine due its potential to reduce costs, improve outcomes, and increase access to care ${ }^{7}$. Telemedicine has also been demonstrated to increase healthcare professionals' adherence to established therapeutic measures for STEMI, along with a statistically significant reduction in in-hospital, 30-day, and one-year mortality ${ }^{8}$. We have previously reported on a pilot programme that displayed the economic value of a population-based telemedicine-guided STEMI network in a controlled cohort [Yoculan A, Kim E, Eggington S, Au-Yeung A. PMD26 - Economic Value of STEMI program investment in Sao Paulo, Brazil. Value in Health. 2015;18:A861]. In our present research, we will evaluate further the impact of the said network on STEMI diagnosis, treatment, and outcomes.

\section{Editorial, see page 11}

\section{Methods}

Using established STEMI guidelines, a standardised Latin America Telemedicine Infarct Network (LATIN) protocol (Figure 1) was created to guide comprehensive AMI management. We implemented this protocol in selected regions of Brazil and Colombia, establishing a hub-and-spoke model at each LATIN site. Centres with 24/7 PCI capabilities ("hubs") were identified in each region to perform STEMI interventions for patients referred from smaller clinics or centres ("spokes"). The spokes functioned as the point of first medical contact for patients, where a cardiologist was usually not present. The spokes were small community health and primary care centres with very limited resources, often having a single primary care physician with limited expertise in managing AMI. Three remote telemedicine command centres were established within the programme (Uberlandia and Sao Paulo in Brazil, and Bogota, Colombia), where expert cardiologists provided urgent electrocardiogram (ECG) diagnosis and tele-consultation for the entire network. The role of telemedicine was to connect expert cardiologists, at three remote sites, who were working full time for LATIN to diagnose a STEMI on an ECG, and subsequently trigger the LATIN protocol to transport that patient to a hub so that they could undergo a PCI. Telemedicine was used to transmit a patient's ECG (performed at a "spoke') to the cardiologist, and subsequently to allow that cardiologist to diagnose a STEMI and guide the treatment process, all remotely. Telemedicine platforms installed at these sites provided immediate diagnosis, secure user and network interface, compatibility, quality assurance, and database management. Automatic vectorisation of the ECG with elimination of noise artefacts and incorporation into the medical record was utilised. Communication software generated automatic alerts from the remote cardiologist to both the hubs and spokes in addition to providing routine feedback. All ECGs that were performed at the spokes were remotely sent to the telemedicine centre. After processing of those ECGs by technicians (including inputting patient demographics and symptoms), all were read by a LATIN cardiologist. ECGs labelled high-risk for STEMI were prioritised. These remote cardiologists were responsible for triggering ambulance dispatch and initiation of the standardised LATIN protocols.

Hub-and-spoke configurations are shown in Figure 2. The comprehensive LATIN strategy and standardised protocol are depicted in Figure 1. The primary responsibilities of the hubs were to perform STEMI interventions with short D2B times. The spokes (an average of up to 5 per hub), located between 5 and 250 miles from each hub, administered fibrinolytic therapy to eligible patients and expedited transfer to the hubs for those eligible for PCI. This time period is quantified as first medical contact to intervention. Each LATIN spoke had advanced directives for treating AMI patients with either primary PCI or fibrinolysis based on the duration of chest pain and their location and driving distance to the hub. Fibrinolytic therapy was recommended for early presentation ( $<3$ hours from onset of pain), while PCI was advocated when transfer to a hub was readily available. Failed fibrinolysis provided an absolute indication for transfer to the hub for rescue PCI. All patients with successful fibrinolysis were transferred to the hub within 4-24 hours. The time to transfer was factored when considering the treatment pathways for each patient, with a goal transfer time (TT) of $<60$ minutes for those referred for PCI. With the use of the LATIN system, this allowed the majority of patients with STEMI to meet the criteria for reperfusion therapy using PCI, with a D2B of $<90$ minutes. The significant transition from fibrinolysis to PCI for the treatment of STEMIs is credited to the 


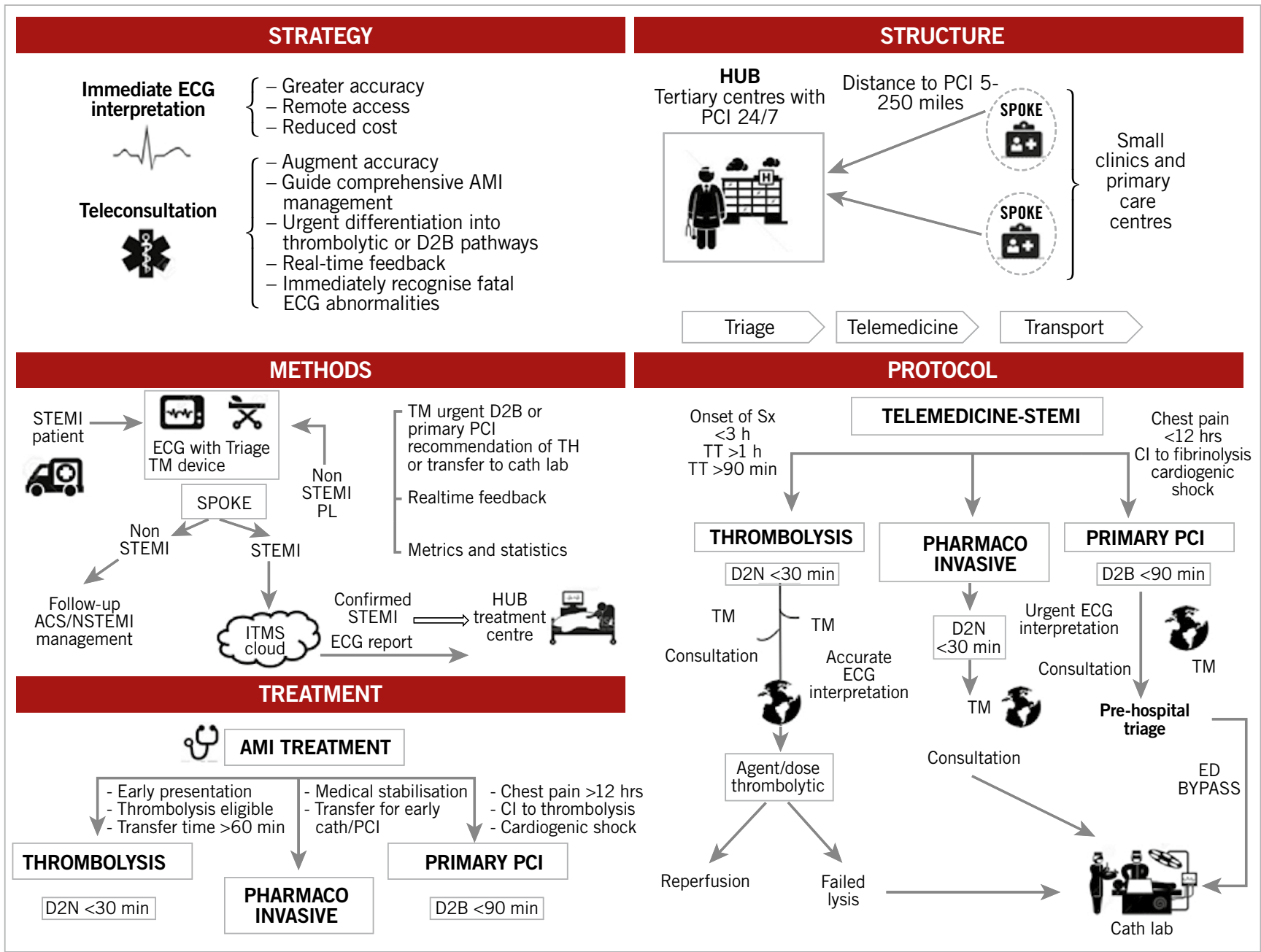

Figure 1. Comprehensive LATIN strategy and standardised protocol. CI: contraindication; D2B: door to balloon time; D2N: door to needle time; ECG: electrocardiogram; ITMS: International Telemedical Systems; PCI: percutaneous coronary intervention; STEMI: ST-elevation myocardial infarction; TH: telemedicine hub; TM: telemedicine device; TT: transfer time

efficiency with which LATIN operates. The LATIN database provided capture of important metrics to measure individually and benchmark all three essential components of the LATIN pathway - spokes, hubs, and telemedicine systems. With this methodology, we employed door in and door out time (DIDO) and mean TT to measure spoke functionality. D2B times gauged hub performance; time to telemedicine diagnosis (TTD) quantified telemedicine efficiency.
Individual patient data from the LATIN site at Santa Marcelina Hospital were compared with health statistics obtained from the Brazilian Public Health System. Both facilities are located in Sao Paulo, Brazil. We also compared data obtained from sites in Brazil versus Colombia, and provided a comparison between health care in the USA and the countries where LATIN operates. The sets of data (Table 1-Table 5) collected were defined by timeframes relative to the implementation of the LATIN protocol - before

Table 1. Demographics: age, gender, before and after implementation of LATIN.

\begin{tabular}{|l|c|c|c|c|c|c|c|c|}
\hline \multirow{2}{*}{} & \multicolumn{3}{|c|}{ Male } & \multicolumn{2}{c|}{ Female } & \multicolumn{2}{c|}{ Age } \\
\cline { 2 - 11 } & Before & After & Before & After & p-value & Before & After & p-value \\
\hline CABG & 36 & 46 & 20 & 24 & 0.867 & 62.3 & 63.9 & 0.294 \\
\hline PCI & 397 & 510 & 227 & 313 & 0.0001 & 60.5 & 61.4 & 0.311 \\
\hline Medical management & 168 & 154 & 120 & 97 & 0.475 & 63.2 & 63.3 & 0.126 \\
\hline Total & 658 & 748 & 404 & 41 & 0.0001 & & & \\
\hline CABG: coronary artery bypass graft; PCl: percutaneous coronary intervention & & &
\end{tabular}




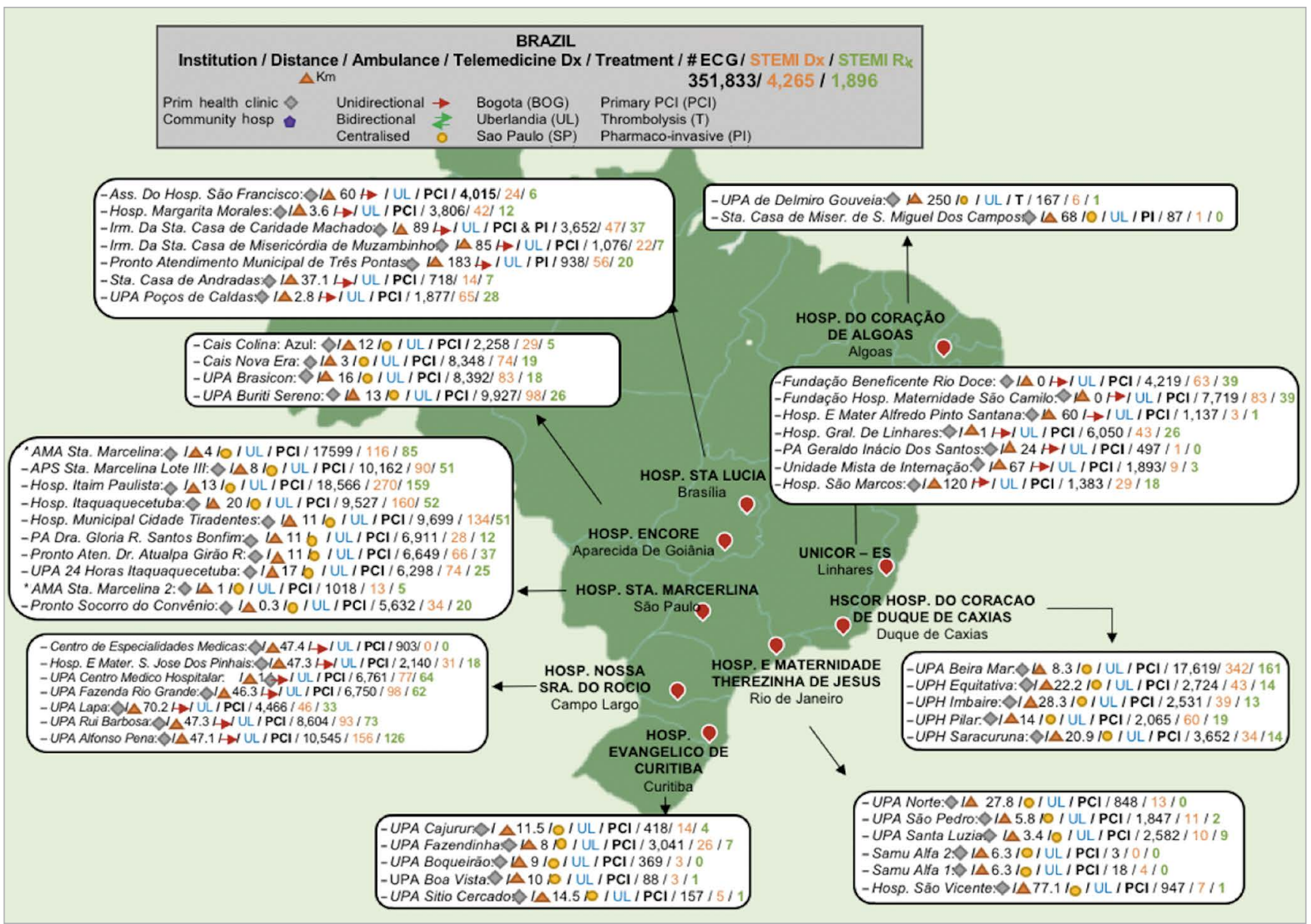

Figure 2. LATIN telemedicine systems of care + hub-and-spoke configuration. BOG: Bogota; PCI: percutaneous coronary intervention; PI: pharmaco-invasive; SP: Sao Paulo; T: thrombolysis; UL: Uberlandia

Table 2. Impact of LATIN on STEMI treatment strategy.

\begin{tabular}{|l|c|c|c|}
\hline & $\begin{array}{c}\text { Pre-LATIN } \\
\text { strategy } \\
(\mathbf{n = 1 , 0 9 5 )}\end{array}$ & $\begin{array}{c}\text { LATIN } \\
\text { strategy } \\
(\mathbf{n = 1 , 2 4 7 )}\end{array}$ & $\boldsymbol{p}$-value \\
\hline CABG & $5.79 \%$ & $6.17 \%$ & 0.736 \\
\hline PCI & $65.27 \%$ & $75.52 \%$ & $<0.001$ \\
\hline $\begin{array}{l}\text { Medical } \\
\text { management }\end{array}$ & $28.66 \%$ & $21.29 \%$ & $<0.001$ \\
\hline Total & $100.00 \%$ & $100.00 \%$ & $<0.738$ \\
\hline $\begin{array}{l}\text { CABG: coronary artery bypass graft; PCl: percutaneous coronary } \\
\text { intervention }\end{array}$ &
\end{tabular}

$\mathrm{n}=1,015$ (October 2012 to March 2014), and after $\mathrm{n}=1,247$ (April 2014 to September 2015). Data collection was focused on mortality, treatment type, and cost, including patient demographics, STEMI intervention, reimbursement fees received for STEMI procedures, and in-hospital mortality. We tested for differences (before versus after implementation of the LATIN protocol) in the mean procedure cost (using the Mann-Whitney $U$ test due to the presence of skewness in the cost data), the mortality rate (by procedure type and overall, using a Z-test for two proportions), and in the procedure mix (using the Z-test). All statistical analyses were performed in Minitab ${ }^{\circledR} 17.2$ (State College, PA, USA).

Table 3. Mortality statistics before and after implementation of LATIN.

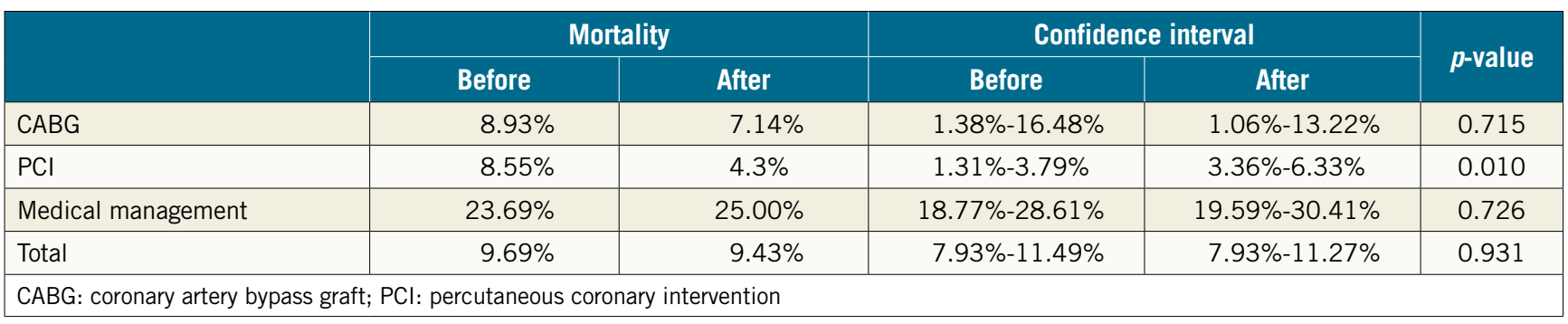


Table 4. Economic impact on STEMI treatment before and after implementation of LATIN.

\begin{tabular}{|l|c|c|c|c|c|}
\hline \multirow{2}{*}{} & \multicolumn{2}{|c|}{ Mean } & \multicolumn{2}{c|}{ Median } & \multicolumn{1}{c|}{ A-value } \\
\cline { 2 - 5 } & Before & After & Before & $\$ 5,369.92$ \\
\hline CABG & $\$ 5,688.64$ & $\$ 5,657.28$ & $\$ 5,472.64$ & 0.4466 \\
\hline PCl & $\$ 2,391.04$ & $\$ 2,503.36$ & $\$ 2,018.88$ & $\$ 2,050.88$ & 0.4181 \\
\hline Medical management & $\$ 628.16$ & $\$ 649.62$ & $\$ 223.04$ & $\$ 220.48$ & 0.001 \\
\hline Total & $\$ 2,037.12$ & $\$ 2,246.40$ & $\$ 1,858.88$ & $\$ 1,949.44$ & 0.0048 \\
\hline *Conversion rate: 1 Brazilian real=0.32 United States dollar. CABG: coronary artery bypass graft; PCl: percutaneous coronary intervention \\
\hline
\end{tabular}

Table 5. Economic and healthcare disparities between developed and developing countries.

\begin{tabular}{|c|c|c|c|}
\hline Indicator & USA & Brazil & Colombia \\
\hline Population, millions & 323.1 & 207.7 & 48.6 \\
\hline Urban population, \% & 82.5 & 86 & 77.4 \\
\hline Per capita income, USD & 57,220 & 15,646 & 14,164 \\
\hline $\begin{array}{l}\text { Annual national health } \\
\text { expenditure as a proportion of } \\
\text { GDP, \% }\end{array}$ & 17.1 & 8.3 & 7.2 \\
\hline $\begin{array}{l}\text { Total health expenditure per } \\
\text { capita, USD }\end{array}$ & 9,403 & 1,318 & 962 \\
\hline $\begin{array}{l}\text { Poverty head count ratio at } \$ 3.1 \\
\text { a day PPP, \% }\end{array}$ & - & 7.6 & 13.2 \\
\hline $\begin{array}{l}\text { Physician ratio (per 100,000 } \\
\text { Pop.) }\end{array}$ & 255 & 185 & 156 \\
\hline $\begin{array}{l}\text { Cardiologist ratio (per 100,000 } \\
\text { Pop.) }\end{array}$ & 31.5 & 13.4 & 7.1 \\
\hline $\begin{array}{l}\text { Hospital beds ratio (per } 100,000 \\
\text { Pop.) }\end{array}$ & 326 & 230 & 160 \\
\hline Total number of cardiac cath labs & 2,000 & 799 & 245 \\
\hline Total PCI/year & 400,000 & 150,000 & 45,000 \\
\hline $\begin{array}{l}\text { Total number of ambulances (per } \\
100,000 \text { Pop.) }\end{array}$ & 48.3 & 10.9 & 4.8 \\
\hline $\begin{array}{l}\text { Ischaemic heart disease mortality } \\
\text { rate (per 100,000 Pop.) }\end{array}$ & 114.8 & 114.1 & 80.5 \\
\hline \multicolumn{4}{|c|}{ Estimated mortality rate for ischaemic heart disease } \\
\hline $45-64$ years & 85.38 & 102.40 & 103.01 \\
\hline$>65$ years & 749.55 & 518.77 & $1,008.30$ \\
\hline \multicolumn{4}{|c|}{$\begin{array}{l}\text { GDP: gross domestic product; PCI: percutaneous coronary intervention; } \\
\text { Pop: population; USA: United States of America; USD: United States } \\
\text { dollars }\end{array}$} \\
\hline
\end{tabular}

\section{Results}

Figure 3 depicts the management of LATIN patients beginning with the screening ECG and ending with definitive STEMI treatment. Between April 2014 and August 2018, 313,897 patients (Brazil 224,423, 71.5\%; Colombia 89,474, 28.5\%) were screened with an ECG, with 3,572 (1.1\%) meeting STEMI criteria. Their telemedicine diagnosis prompted a simultaneous telemedicine alert to both the hub and spoke. A total of 1,636 patients $(45.8 \%$ of STEMI patients, $0.52 \%$ of the screened cohort) underwent urgent treatment via triggering of the LATIN protocol.

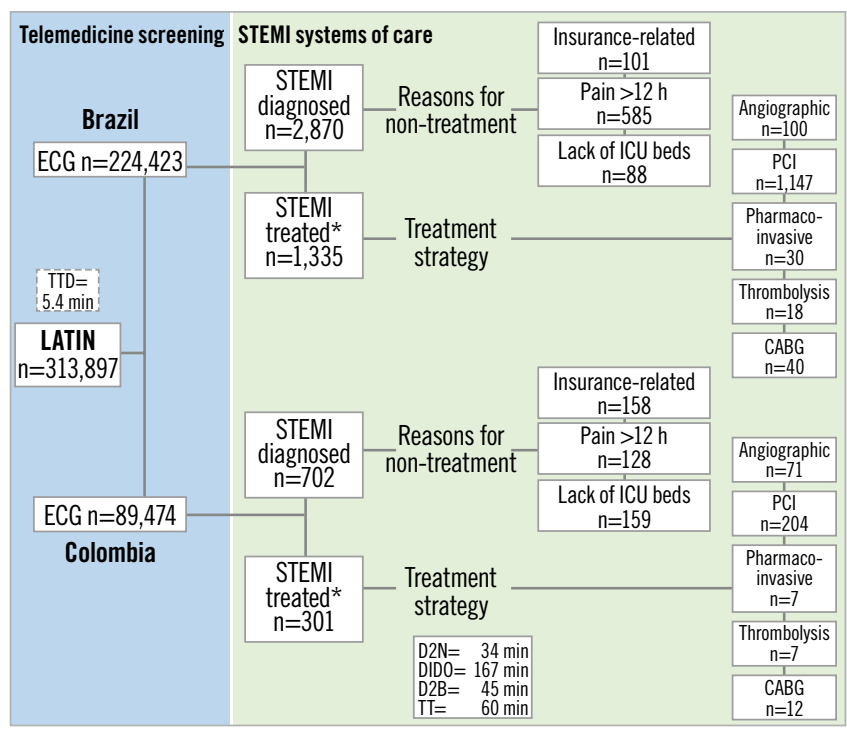

Figure 3. LATIN flow chart. CABG: coronary artery bypass graft; D2B: door to balloon time; D2N: door to needle; DIDO: door in door out; ECG: electrocardiogram; ICU: intensive care unit; PCI: percutaneous coronary intervention; STEMI: ST-elevation myocardial infarction; TTD: time to telemedicine diagnosis

Primary PCI $1,351(82.6 \%)$, coronary artery bypass graft (CABG) 52 (3.2\%), pharmaco-invasive 37 (2.3\%), and fibrinolysis $25(1.5 \%)$ were the methods of reperfusion in the $1,636(48.5 \%)$ of 3,572 telemedicine-diagnosed STEMI. Average door to needle time (D2N) was 81.3 minutes, D2B 51.3 minutes, DIDO $106.3 \mathrm{~min}$ and TT 60 minutes. Mean TTD was 5.4 minutes (range 3-7 minutes).

For LATIN patients, total in-hospital mortality was 183 patients (5.1\%); the hub-and-spoke breakdown included 87 deaths at the spokes (47.8\%), 29 during transfer $(15.8 \%)$ and 67 at the hubs (36.4\%). The mortality breakdown for CABG, PCI, and medical management pre versus post LATIN is as follows: CABG $(8.93 \%$, confidence interval [CI]: $1.38-16.48$, vs $7.14 \%$, CI: $1.06-13.22$, $\mathrm{p}=0.715)$; for PCI $(8.55 \%$, CI: $1.31-3.79$, vs $4.3 \%$, CI: $3.36-6.33$, $\mathrm{p}<0.01)$; for medical management (23.69\%, CI: 18.77-28.61, $\mathrm{p}=0.726$ ). Overall mortality tended to be lower for the LATIN group but was not statistically significant (9.43\%, CI: 7.93-11.27, vs $9.69 \%$, CI: 7.93-11.49, $\mathrm{p}=0.931)$. Medical management $(26.2 \%$ vs $20.0 \%, \mathrm{p}<0.001$ ) was performed in a significantly larger number of pre-LATIN patients. The number of patients treated with PCI was significantly higher for the LATIN group $(68.1 \%$ vs 
$59.9 \%, \mathrm{p}<0.001)$. Both groups had similar rates of CABG $(5.8 \%$ vs $5.3 \%, \mathrm{p}=0.736$ ).

Overall, LATIN management demonstrated an increase in the cost of treatment (median, $\$ 1,949.44$ vs $\$ 1,858.88$, $p<0.0048$ ). For the treatment groups, the costs were as follows: CABG, median $\$ 5,369.92$ vs $\$ 5,472.64, \mathrm{p}=0.4466$; PCI, median $\$ 2,050.88$ vs $\$ 2,018.88, \mathrm{p}=0.4181$; medical management, median $\$ 220.48$ vs $\$ 223.04, \mathrm{p}<0.001$.

\section{Discussion}

The risk associated with the fact that STEMI is initially misdiagnosed in nearly a third of patients remains a fundamental process issue in primary $\mathrm{PCI}^{9,10}$. Computerised interpretation of the ECG alone cannot be relied upon and requires confirmation by an experienced ECG reader, thus the use of cardiologists is an important part of LATIN's efficiency ${ }^{10}$. Digitisation ${ }^{11}$ and telemedicine provide potential solutions to this challenging problem ${ }^{7,12}$.

Using telemedicine, a standardised guideline-based protocol, and a hub-and-spoke strategy, LATIN established a multi-nation STEMI network. LATIN remotely guides STEMI diagnosis and management while simultaneously overcoming geographic barriers. Researchers have demonstrated a reduction in ischaemic time for transferred STEMI patients using a smartphone network $^{13}$ and cloud computing ${ }^{14}$. The number of patients requiring pre-hospital triage has been nearly halved with telemedicine use; the most improvement was seen in populations with longer delays to treatment ${ }^{15}$. By increasing access to comprehensive STEMI care, LATIN reduces disparities of AMI care that exist between developed and developing countries ${ }^{16}$, [Mehta S, Ferre A, Botelho R, Fernandes F, Vega R, Bojanini F, Corral J, Rodriguez D, Cardoso R, Perin M, Campos C. 4143 Telemedicine increases access, accuracy \& cost-effectiveness of population based AMI care: results of the Latin America telemedicine infarct network (LATIN). Eur Heart J. 2017;38: ehx504.4143.] [Dallan L, Pazolini V, Matsuda C, Cintra G, Opazo C, Janella B, Cade J, Botelho R, Mehta S, Perin MA. CRT-124 Telemedicine as a landmark in the reduction of the door-to-balloon time in STEMIs in distant areas in a developing country. JACC Cardiovasc Interv. 2015;8(2_S):S15-S15.]

It can also serve as a model to overcome barriers of care in other areas with similar logistical, infrastructure, and economic constraints [Mehta S, Ferre A, Botelho R, Fernandes F, Vega R, Bojanini F, Corral J, Rodriguez D, Cardoso R, Perin M, Campos C. 4143 Telemedicine increases access, accuracy \& cost-effectiveness of population based AMI care: results of the Latin America telemedicine infarct network (LATIN). Eur Heart J. 2017;38: ehx504.4143.] [Mehta S, Ferre A, Botelho R, Fernandes F, Vega R, Bojanini F, Corral J, Rodriguez D, Cardoso R, Perin M, Campos C. P3408 Telemedicine + STEMI systems of care: novel solution to massively expand access to AMI care in developing countries. Eur Heart J. 2017;38:ehx504.P3408.] Numerous other researchers have exploited additional benefits of telemedicine and there has been a proliferation of telemedicine-guided STEMI networks for both public and private hospitals ${ }^{17}$, particularly in large communities in Sao Paulo, Brazil, and in Warsaw, Poland ${ }^{18}$ [Matsuda C, Cade J, Janella B. Implementação do Sistema de Telemedicina no Atendimento Inicial dos Pacientes com Infarto Agudo do Miocárdio com Supradesnivelamento do Segmento ST na Zona Leste da Cidade de São Paulo. Paper presented at: 40th Congress of the Brazilian Society of Hemodynamics and Interventional Cardiology; June 2015; Brasilia, Brazil].

LATIN demonstrated a sharp shift from the earlier pattern, of predominant fibrinolysis or no reperfusion, to significantly higher rates of reperfusion with PCI, which was also the group with the lowest mortality rate (Table 3 ). The shift of care to a greater utilisation of PCI did incur a higher cost, although it is possible that this higher initial cost will be negated by the longer-term benefits of earlier return to work and decreased disability. This was also seen when investigators evaluating the efficiency of a STEMI network developed to treat the 7.5 million inhabitants of Catalonia (Codi Infarct) demonstrated an aggregate cost increase of $2.6 \%$ with a reduction in mortality from $7.5 \%$ to $5.6 \%{ }^{19}$.

The LATIN cohort treated with fibrinolysis is much lower than that in various other trials. Mostly, this was by intent, as it was observed that both Brazil and Colombia were relying heavily on first-generation fibrinolytics despite patients' presentation beyond the ideal timeframe for effective fibrinolysis. LATIN's scrupulous attention to ambulance transfer is reflected in D2N and D2B times. Reliable transportation ensured that PCI could be performed at most sites in a timely fashion. Prolonged D2N time probably resulted from this being an unpopular strategy and from its relative underutilisation. The American Heart Association, American College of Cardiology, and European Society of Cardiology have guidelines strongly advocating a D2N time of less than 30 minutes when a fibrinolytic strategy is employed ${ }^{19}$ [Reilly NM, Gunnarsson SI, Ciske B, Saari S, Leal M, Raval A, O'Connor A. Abstract 16112: In Hospital Triage of ST Segment Myocardial Infarction Patients Results in Favorable Clinical Outcomes and Reduced ICU and Overall Hospital Length of Stay. Circulation. 2016;134:A16112.]. A short D2B (under 90 minutes) has become the mantra for primary PCI, with a direct correlation between short D2B and both early and long-term mortality ${ }^{21}$. Current guidelines direct the pharmaco-invasive strategy beginning with fibrinolysis for patients for whom primary PCI cannot be provided in a timely fashion $^{1,2,22}$. Despite the considerable progress, self-transportation, as opposed to ambulance transfer, is still widespread and is an independent predictor of inpatient mortality ${ }^{23}$. However, when training and experience are properly utilised in STEMI systems of care, patients presenting off-hours have similar short-, intermediate-, and long-term results relative to patients presenting during on-hours, with no difference in $\mathrm{D}_{2} \mathrm{~B}^{24}$. Furthermore, we anticipate that this network will be useful for administering cardioprotective drugs such as fingolimod and exenatide, which have been demonstrated to reduce myocardial ischaemia-reperfusion injury and increase myocardial salvage ${ }^{25,26}$. Although long-term outcomes have not been measured for use in this manuscript, it is likely that 
the inclusion of these cardioprotective medications in the LATIN protocol would provide a significant long-term mortality benefit.

Improving patient outcomes and efficiency was guided by a "3Ts strategy", efficient triage, telemedicine, and transport at each spoke in addition to $24 / 7$ primary PCI capability at each hub. With an incrementally paced training process and this 3Ts strategy, we saw improvement in the process parameters of DIDO and TT. The small size of LATIN hub hospitals allowed emergency department (ED) bypass to occur without delay which contributed significantly to reduced D2B times. Single page activation, strict on-call schedules, mandated reporting times, effective communication, teamwork, and continuous feedback also contributed to hub efficiency. A combination of efforts at both spokes and hubs has created a persuasive trend in reduced PCI mortality in both Brazil and Colombia.

\section{Limitations}

Our current research has not demonstrated an overall reduction in mortality across all treatment pathways. It is possible that statistically significant mortality differences can be established with data analyses of subsets of LATIN patients including male versus female, those at higher risk for AMI by typical risk factors, and with age stratification. In Apulia, Italy, Brunetti et al created a pre-hospital ECG diagnosis network that has shown augmented STEMI diagnosis and cost-effectiveness with more selective patient criteria ${ }^{15}$. We are actively redesigning our screening process and adjusting inclusion criteria for coronary risk factors. With the expansion of LATIN sites into other parts of Central and South America, the screening process will integrate criteria more sensitive for AMI.

LATIN encountered numerous system deficits that were stubbornly resistant to improvement and may have diminished the overall impact of the AMI programme. The large proportion of non-treated patients has also diminished the cost-effectiveness of LATIN and the overall screening power of telemedicine. Amongst the numerous reasons accounting for lack of STEMI treatment in 1,936 patients, the three most common were chest pain $>12$ hours (713; 36.8\%), lack of intensive care unit (ICU) beds (247; 12.75\%), and insurance denials $(259 ; 13.37 \%)$. Both countries demonstrated similar rates of insurance denials. Colombia was constrained more by lack of beds, whereas delayed presentation was more common in Brazil. The transfer of a post-PCI patient to a non-ICU bed has demonstrated some success and is currently being pursued as a solution. With increased LATIN use and population education, we hope to see significant improvements in the number of diagnosed STEMI patients receiving treatment.

The only public health data available for statistical analysis were from the Sistema Unico de Saude (SUS), and now LATIN. The fact that data were gathered from different sites allowed significant confounders to occur in the data collected. However, this allows us to identify the impact on management for AMI with the use of LATIN. Our research did not explore the long-term effects of a telemedicine strategy. Additionally, a single-centre analysis may not be generalisable to all LATIN sites. The absence of existing data prior to the establishment of LATIN caused us to rely more on prospective analysis. As LATIN continues to expand into other nations, and the number of patients screened and treated increases, we plan on continuing data collection and analysis to track and gauge changes in outcomes.

\section{Conclusions}

The Latin America Telemedicine Infarct Network (LATIN) uses a hub-and-spoke model, guided by remote cardiologists, to diagnose and guide management of acute myocardial infarction. The significance of this network is the increased access to standard of care treatment in developing regions where health care normally would not reach. We have significantly increased the proportion of patients receiving PCI for STEMI treatment, with a significant reduction in the mortality of those undergoing PCI. Telemedicine increases access to care and improves mortality and outcomes. Networks such as LATIN are especially relevant and necessary in underdeveloped and underserved regions of the world given the increasing global burden of cardiovascular disease (CVD) on morbidity and mortality.

\section{Impact on daily practice}

PCI continues to be the standard of care for management of STEMI/AMI. Efforts to expand evidence-based medical care to all patients including those socioeconomically disadvantaged and in underserved regions are prudent, given the clear mortality benefit, global burden of CVD, and responsibility for judicious allocation of resources and equity in health care. Physicians continue to be the expert clinicians and leaders in the development of standard of care and its implementation. Telemedicine is asserting itself as a viable resource to increase the outreach of physician expertise and leadership to large patient populations while maintaining superior outcomes.

\section{Funding}

Education grant from the Medtronic Foundation (Santa Rosa, CA, USA).

\section{Conflict of interest statement}

The authors have no conflicts of interest to declare.

\section{References}

1. Levine GN, Bates ER, Blankenship JC, Bailey SR, Bittl JA, Cercek B, Chambers CE, Ellis SG, Guyton RA, Hollenberg SM, Khot UN, Lange RA, Mauri L, Mehran R, Moussa ID, Mukherjee D, Ting HH, O'Gara PT, Kushner FG, Ascheim DD, Brindis RG, Casey DE Jr, Chung MK, de Lemos JA, Diercks DB, Fang JC, Franklin BA, Granger CB, Krumholz HM, Linderbaum JA, Morrow DA, Newby LK, Ornato JP, Ou N, Radford MJ, TamisHolland JE, Tommaso CL, Tracy CM, Woo YJ, Zhao DX. 2015 ACC/AHA/SCAI Focused Update on Primary Percutaneous Coronary Intervention for Patients With ST-Elevation Myocardial Infarction: An Update of the 2011 ACCF/AHA/SCAI Guideline for 
Percutaneous Coronary Intervention and the 2013 ACCF/AHA Guideline for the Management of ST-Elevation Myocardial Infarction: A Report of the American College of Cardiology/American Heart Association Task Force on Clinical Practice Guidelines and the Society for Cardiovascular Angiography and Interventions. Circulation. 2016;133:1135-47.

2. Ibanez B, James S, Agewall S, Antunes MJ, Bucciarelli-Ducci C, Bueno H, Caforio ALP, Crea F, Goudevenos JA, Halvorsen S, Hindricks G, Kastrati A, Lenzen MJ, Prescott E, Roffi M, Valgimigli M, Varenhorst C, Vranckx P, Widimský P; ESC Scientific Document Group. 2017 ESC Guidelines for the management of acute myocardial infarction in patients presenting with ST-segment elevation: The Task Force for the management of acute myocardial infarction in patients presenting with ST-segment elevation of the European Society of Cardiology (ESC). Eur Heart J. 2018; 39:119-77.

3. Oldroyd KG. Complete Immediate Revascularization of the Patient With ST-Segment-Elevation Myocardial Infarction Is the New Standard of Care. Circulation. 2017;135:1571-3.

4. Acharya T, Salisbury AC, Spertus JA, Kennedy KF, Bhullar A, Reddy HKK, Joshi BK, Ambrose JA. In-Hospital Outcomes of Percutaneous Coronary Intervention in America's Safety Net: Insights From the NCDR Cath-PCI Registry. JACC Cardiovasc Interv. 2017;10:1475-85.

5. Mentias A, Raza MQ, Barakat AF, Youssef D, Raymond R, Menon V, Simpfendorfer C, Franco I, Ellis S, Tuzcu EM, Kapadia SR. Effect of Shorter Door-to-Balloon Times Over 20 Years on Outcomes of Patients With Anterior ST-Elevation Myocardial Infarction Undergoing Primary Percutaneous Coronary Intervention. Am J Cardiol. 2017;120:1254-9.

6. Prasad A, Gersh BJ, Mehran R, Brodie BR, Brener SJ, Dizon JM, Lansky AJ, Witzenbichler B, Kornowski R, Guagliumi G, Dudek D, Stone GW. Effect of Ischemia Duration and Door-to-Balloon Time on Myocardial Perfusion in ST-Segment Elevation Myocardial Infarction: An Analysis From HORIZONS-AMI Trial (Harmonizing Outcomes with Revascularization and Stents in Acute Myocardial Infarction). JACC Cardiovasc Interv. 2015;8:1966-74.

7. Daniel H, Sulmasy LS; Health and Public Policy Committee of the American College of Physicians. Policy recommendations to guide the use of telemedicine in primary care settings: an American College of Physicians position paper. Ann Intern Med. 2015; 163:787-9.

8. Marcolino MS, Maia LM, Oliveira JAQ, Melo LDR, Pereira BLD, Andrade-Junior DF, Boersma E, Ribeiro AL. Impact of telemedicine interventions on mortality in patients with acute myocardial infarction: a systematic review and meta-analysis. Heart. 2019; 105:1479-86.

9. Mayor S. Acute MI is initially misdiagnosed in nearly a third of patients, study finds. BMJ. 2016;354:i4713.

10. Schläpfer J, Wellens HJ. Computer-interpreted Electrocardiograms: Benefits and Limitations. J Am Coll Cardiol. 2017;70:1183-92.

11. Steinhubl SR, Topol EJ. Moving From Digitalization to Digitization in Cardiovascular Care: Why is it Important, and What
Could it Mean for Patients and Providers? J Am Coll Cardiol. 2015; 66:1489-96.

12. Madder RD, VanOosterhout SM, Jacoby ME, Collins JS, Borgman AS, Mulder AN, Elmore MA, Campbell JL, McNamara RF, Wohns DH. Percutaneous coronary intervention using a combination of robotics and telecommunications by an operator in a separate physical location from the patient: an early exploration into the feasibility of telestenting (the REMOTE-PCI study). EuroIntervention. 2017;12:1569-76.

13. Park JJ, Yoon CH, Suh JW, Cho YS, Youn TJ, Chae IH, Choi DJ. Reduction of Ischemic Time for Transferred STEMI Patients Using a Smartphone Social Network System. J Am Coll Cardiol. 2016; 68:1490-2.

14. Ho CK, Chen FC, Chen YL, Wang HT, Lee CH, Chung WJ, Lin CJ, Hsueh SK, Hung SC, Wu KH, Liu CF, Kung CT, Cheng CI. Using a Cloud Computing System to Reduce Door-to-Balloon Time in Acute ST-Elevation Myocardial Infarction Patients Transferred for Percutaneous Coronary Intervention. Biomed Res Int. 2017;2017:2963172.

15. Brunetti ND, De Gennaro L, Correale M, Santoro F, Caldarola P, Gaglione A, Di Biase M. Pre-hospital electrocardiogram triage with telemedicine near halves time to treatment in STEMI: A meta-analysis and meta-regression analysis of non-randomized studies. Int $J$ Cardiol. 2017;232:5-11.

16. Tanguay A, Dallaire R, Hébert D, Bégin F, Fleet R. Rural Patient Access to Primary Percutaneous Coronary Intervention Centers is improved by a Novel Integrated Telemedicine Prehospital System. J Emerg Med. 2015;49:657-64.

17. Macedo TA, de Barros E Silva PG, Simões SA, Okada MY, Garcia JC, Sampaio MC, Dantas RN Jr, Oliveira RP, Rocha LG, da Cunha Lopes BB, Frigini T, Furlan V. Impact of Chest Pain Protocol with Access to Telemedicine on Implementation of Pharmacoinvasive Strategy in a Private Hospital Network. Telemed $J E$ Health. 2016;22:549-52.

18. Rekosz J, Kasznicka M, Kwiatkowska D, MączyńskaMazuruk R, Kołak K, Mierzejewska B, Drogosiewicz D, Timler D, Gałązkowski R. Standard 12-lead electrocardiogram tele-transmission: Support in diagnosing cardiovascular diseases in operations undertaken by Warsaw-area basic medical rescue teams between 2009 and 2013. Cardiol J. 2015;22:675-82.

19. Regueiro A, Bosch J, Martín-Yuste V, Rosas A, Faixedas MT, Gómez-Hospital JA, Figueras J, Curós A, Cequier A, Goicolea J, Fernández-Ortiz A, Macaya C, Tresserras R, Pellisé L, Sabaté M. Cost-effectiveness of a European ST-segment elevation myocardial infarction network: results from the Catalan Codi Infarct network. BMJ Open. 2015;5:e009148.

20. Jordan M, Caesar J. Improving door-to-needle times for patients presenting with ST-elevation myocardial infarction at a rural district general hospital. BMJ Qual Improv Rep. 2016;5:u209049w6736.

21. Kutcher MA. Door-to-Balloon Time as a Process Metric for Treatment of ST-Segment Elevation Myocardial Infarction: Time to “Tap Out”? JACC Cardiovasc Interv. 2015;8:1975-7. 
22. Patel N, Patel NJ, Thakkar B, Singh V, Arora S, Patel N, Savani C, Deshmukh A, Thadani U, Badheka AO, Alfonso C, Fonarow GC, Cohen MG. Management Strategies and Outcomes of ST-Segment Elevation Myocardial Infarction Patients Transferred After Receiving Fibrinolytic Therapy in the United States. Clin Cardiol. 2016;39:9-18.

23. Kodama N, Nakamura T, Yanishi K, Nakanishi N, Zen K, Yamano T, Shiraishi H, Shirayama T, Shiraishi J, Sawada T, Kohno Y, Kitamura M, Furukawa K, Matoba S. Impact of Door-toBalloon Time in Patients With ST-Elevation Myocardial Infarction Who Arrived by Self-Transport - Acute Myocardial InfarctionKyoto Multi-Center Risk Study Group. Circ J. 2017;81:1693-8.

24. Enezate TH, Omran J, Al-Dadah AS, Alpert M, Mahmud E, Patel M, Aronow HD, Bhatt DL. Comparison of Outcomes of STElevation Myocardial Infarction Treated by Percutaneous Coronary
Intervention During Off-Hours Versus On-Hours. Am J Cardiol. 2017;120:1742-54.

25. Santos-Gallego CG, Vahl TP, Goliasch G, Picatoste B, Arias T, Ishikawa K, Njerve IU, Sanz J, Narula J, Sengupta PP, Hajjar RJ, Fuster V, Badimon JJ. Sphingosine-1 Phosphate Receptor Agonist Fingolimod Increases Myocardial Salvage and Decreases Adverse Postinfarction left Ventricular Remodeling in a Porcine Model of Ischemia/Reperfusion. Circulation. 2016; 133:954-66.

26. Lonborg J, Vejlstrup N, Kelbaek H, Botker H, Kim WY, Mathiasen A, Jorgensen E, Helqvist S, Saunamaki K, Clemmensen P, Holmvang L, Thuesen L, Krusell LR, Jensen JS, Køber L, Treiman M, Holst JJ, Engstrøm T. Exenatide reduces reperfusion injury in patients with ST-segment elevation myocardial infarction. Eur Heart J. 2012;33:1491-9. 